\title{
HepaCAM inhibits cell proliferation and invasion in prostate cancer by suppressing nuclear translocation of the androgen receptor via its cytoplasmic domain
}

\author{
QINGFU DENG ${ }^{1}, \mathrm{LI} \mathrm{LUO}^{2}$, ZHEN QUAN $^{1}$, NANJING LIU ${ }^{2}$, ZHONGBO DU $^{1}$, \\ WEI SUN ${ }^{1}$, CHUNLI LUO $^{2}$ and XIAOHOU WU ${ }^{1}$ \\ ${ }^{1}$ Department of Urology, First Affiliated Hospital of Chongqing Medical University; ${ }^{2}$ Key Laboratory of Diagnostics Medicine \\ Designated by The Ministry of Education, Chongqing Medical University, Chongqing 400042, P.R. China
}

Received May 23, 2018; Accepted December 12, 2018

DOI: $10.3892 / \mathrm{mmr} .2019 .9841$

\begin{abstract}
Hepatocyte cell adhesion molecule (HepaCAM) is a tumour suppressor. However, the mechanism of HepaCAM function in prostate cancer $(\mathrm{PCa})$ remains unknown. In the present study, HepaCAM, androgen receptor (AR) and Ran were analysed in 46 PCa tissue samples using immunohistochemistry. Subsequently, the influence of HepaCAM and its cytoplasmic domain on cell proliferation, migration, and invasion, and associated proteins was examined using MTT, wound healing, Transwell and western blotting assays, respectively. Furthermore, nuclear translocation of AR and Ran was analysed using immunofluorescence and Western blot assays. The results demonstrated that HepaCAM expression was reduced in $\mathrm{PCa}$, and there was an association between downregulation of HepaCAM and changes in the distribution of AR and Ran. Furthermore, HepaCAM, specifically the cytoplasmic domain, was involved in cell proliferation, migration and invasion. Nuclear translocation of AR was dependent on HepaCAM and its cytoplasmic domain. Additionally, HepaCAM suppression of the nuclear translocation of AR occurred via Ran. The results suggest that HepaCAM and its cytoplasmic domain suppress the nuclear translocation of AR via Ran in PCa. The cytoplasmic domain of HepaCAM may serve as a novel target for therapy in $\mathrm{PCa}$.
\end{abstract}

\section{Introduction}

Prostate cancer $(\mathrm{PCa})$ is a common type of malignant tumour in the urinary system of elderly males, and has become a major cause of mortality among urological cancers in the USA and

Correspondence to: Professor Xiaohou $\mathrm{Wu}$, Department of Urology, First Affiliated Hospital of Chongqing Medical University, 1 Youyi Road, Yuzhong, Chongqing 400042, P.R. China

E-mail: 394291654@qq.com

Key words: hepatocyte cell adhesion molecules, prostate cancer, androgen receptor, ran, nuclear translocation other industrialized countries (1). Therefore, it is necessary to elucidate the underlying pathophysiological processes of $\mathrm{PCa}$ with the advent of global ageing.

Hepatocyte cell adhesion molecule (HepaCAM) was first detected in the liver (2), and it was later identified as a member of the immunoglobulin superfamily. Members of the immunoglobulin superfamily are primarily localized at the cell membrane and are composed of three domains: Extracellular, transmembrane and cytoplasmic (3-5). The cytoplasmic domain is fundamental to its biological function (2). Recent studies have indicated that HepaCAM is present at low levels or is even absent in certain types of cancer tissues and cell lines $(2,3,6)$. Our previous studies demonstrated that cell proliferation is significantly inhibited when HepaCAM is overexpressed in urological cancer cells (3-5,7). Furthermore, recent studies have revealed that HepaCAM can promote apoptosis and inhibit invasion and migration of cancer cells $(7,8)$. These studies indicate that HepaCAM is a tumour suppressor candidate that may be downregulated in cancer development. However, extensive research is required to elucidate the mechanism of the anti-tumour role of HepaCAM in the carcinogenesis and progression of $\mathrm{PCa}$, particularly that of the cytoplasmic domain of HepaCAM.

Androgen receptor (AR), a steroid hormone receptor, is necessary for the physiological function of the prostate (9) and serves an important role in the proliferation, apoptosis, invasion and metastasis of PCa cells $(10,11)$. After binding to androgens in the cytoplasm, AR undergoes a series of conformational changes to form the AR-androgen complex (12), which translocate from the cytoplasm to the nucleus, and then activates AR-targeted gene expression (13-15). Therefore, investigation of the potential activation mechanisms of AR is crucial in order to identify novel androgen-based treatments for PCa.

Our previous study demonstrated that HepaCAM inhibits the nuclear translocation of the androgen-AR complex (7). Nuclear translocation is a dynamic equilibrium process in which molecular complexes shuttle back and forth through the cell nuclear membrane via nuclear pore complexes (16). Ran proteins serve a key role in providing energy for this process. Ran is a guanine triphosphate (GTP)-binding protein 
that is widely present in the nucleus and circulates between the cytoplasm and the nucleus in the form of RanGDP and RanGTP $(17,18)$. Therefore, it was hypothesized that the anti-tumour effects of HepaCAM may be partially associated with its influence on the AR signalling pathway, which involves AR and Ran. The present study focuses on the biological function of the cytoplasmic domain of HepaCAM and investigates the association among AR, Ran and the cytoplasmic domain of HepaCAM in the process of AR nuclear translocation.

Based on the important role of AR in the progression of $\mathrm{PCa}$, the present study aimed to ascertain whether HepaCAM influenced the endonuclear and extranuclear distribution in PCa tissues and LNCaP cell line, and then to explore the possible mechanisms by which HepaCAM and its cytoplasmic domain affect nuclear translocation of AR. The present study may provide further support for HepaCAM inhibition of $\mathrm{PCa}$.

\section{Materials and methods}

Patients and tissues. A total of 46 PCa specimens from patients who had undergone radical prostatectomy, and 46 benign prostatic hyperplasia (BPH) specimens from patients who had undergone a transurethral resection of the prostate between September 2015 and April 2017 were included. All collected specimens were were fixed, dehydrated, embedded stained with hematoxylin and eosin (H\&E) or immunohistochemical staining (IHC). None of the patients with PCa had undergone androgen deprivation therapy (ADT) or neoadjuvant chemotherapy. All samples were meticulously studied by the same experienced pathologist without misdiagnosis. The stage and Gleason grade of the PCa were diagnosed according to UICC guidelines (19). Informed consent forms were signed by all patients, and the study was approved by the Research Ethical Committee of the Chongqing Medical University (Chongqing, China).

$H \& E$ and IHC. The PCa and BPH tissues were fixed with $10 \%$ neutral formalin for 2 days at room temperature, dehydrated in a graded series of alcohol, embedded in paraffin, and then sectioned with thickness of $4 \mu \mathrm{m}$ for histological examination. H\&E and IHC staining was performed according to a standard procedure (20). The primary antibodies were as follows: Anti-HepaCAM (1:200, ProteinTech Group, Inc., Chicago, IL, USA; cat. no. 18177-1-AP), anti-AR (1:200, ProteinTech Group, Inc.; cat. no. 22576-1-AP), anti-Ran (1:200, Abcam, Cambridge, UK; cat. no. ab157213), horseradish peroxidase-labelled goat anti-rabbit secondary antibody (1:500; OriGene Technologies, Inc., Rockville, MD, USA; cat. no. ZF-0316). The semi-quantitative intensity was defined as follows: 0 (unstained), 1 (yellow), 2 (light brown), and 3 (brown). The immunoreactivity ratio was semi-quantitatively counted as follows: $0(0 \%), 1(<5 \%), 2(5-50 \%)$, and $3(>50 \%)$. The parameters of ratio and intensity were combined to determine the final scores as follows: Samples with score $<3$ were considered negative, and samples with scores $>3$ were regarded as positive for statistical convenience.

Cell culture and transfection. The hormone-sensitive PCa LNCaP cell line was incubated in high-glucose Dulbecco's modified Eagle's medium/F-12 (Gibco; Thermo Fisher
Scientific, Inc., Waltham, MA, USA) supplemented with $100 \mathrm{U} / \mathrm{ml}$ penicillin and streptomycin (Thermo Fisher Scientific, Inc.) at $37^{\circ} \mathrm{C}$ in a $45 \%$ humidified incubator with $5 \% \mathrm{CO}_{2}$. An adenovirus containing intact HepaCAM (ad-HepaCAM) $\left(1 \times 10^{9}\right)$ was constructed by our group as previously described (21). An adenovirus construct with the entire cytoplasmic domain residues (264-416) of HepaCAM deleted

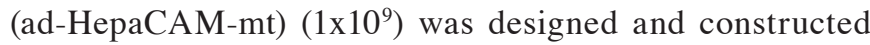
by Shanghai GeneChem Co., Ltd., (Shanghai, China). The cells were incubated firstly in serum-free medium for $24 \mathrm{~h}$ at $37^{\circ} \mathrm{C}$, and then, the adenoviruses described above were used for transfection for $\sim 48 \mathrm{~h}$ at $37^{\circ} \mathrm{C}$ for subsequence analysis. Cells transfected with ad-hepaCAM or ad-hepaCAM-mt were named ad-hepaCAM group or ad-hepaCAM-mt group respectively; the cells transfected with vector that served negative control were named vector group, the cells treated PBS that served blank control were named blank group.

Ran-targeting siRNA (si-Ran) and a scrambled siRNA sequence, which served as negative control (NC) were designed and synthesized by Shanghai GeneChem Co., Ltd. The Ran siRNA sequences was: 5'-CAGAUUGUUCGGUUUGGC UUGUUUA-3', the scrambled siRNA sequences was: 5'-CAG UGUUCGGUUUGGCUUGUAUUUA-3'. When the cell density reached $\sim 30 \%$, si-Ran was transfected into cells using Lipofectamine ${ }^{\circledR} 2000$ (Invitrogen; Thermo Fisher Scientific, Inc.) for about $48 \mathrm{~h}$ at $37^{\circ} \mathrm{C}$ according to the manufacturer's protocol. The cells transfected with si-Ran were named si-Ran group, the cells transfected with ad-hepaCAM and si-Ran were named ad-hepaCAM + si-Ran group. The cells transfected with ad-hepaCAM-mt and si-Ran were named ad-hepaCAM-mt + si-Ran group. To analyze the transfection efficiency, the numbers and staining intensity of positive cells expressing green fluorescent protein (GFP) were observed by fluorescence microscopy at different time points.

MTT assay. Cells (2,000/well) were cultured in 96-wells plates with $100 \mu \mathrm{l}$ of culture medium. After cell attachment was complete, each cell transfection was conducted in five replicate wells At each time point, $5 \mathrm{mg} / \mathrm{ml}$ MTT (Sigma-Aldrich; Merck KGaA, Darmstadt, Germany) was added to the treated wells. Subsequently, the treated cells were maintained for $\sim 3-5 \mathrm{~h}$ at $37^{\circ} \mathrm{C}$. Subsequently, dimethyl sulfoxide was added to each well after removing the culture medium. The 96-well plates were agitated steadily on a rotator platform for $15 \mathrm{~min}$ at room temperature. The absorbance at $490 \mathrm{~nm}$ was recorded on an ultraviolet spectrophotometric reader. The experiments were repeated five times. Wells with treatment-free medium served as the negative control.

Colony formation assay. Cells in each treatment group were seeded in six-well plates at a density of 1,000 cells/well with $3 \mathrm{ml}$ culture medium. After two weeks of incubation, the treated LNCaP cells were washed with PBS twice and then fixed with $4 \%$ paraformaldehyde at $37^{\circ} \mathrm{C}$ for $20 \mathrm{~min}$. The cell clusters were dyed in $0.1 \%$ violet solution for $20 \mathrm{~min}$ at room temperature. The colony numbers were counted under an inverted microscope (Nikon Corporation, Tokyo, Japan). The colony forming efficiency (CFE) was calculated with the following equation: $\mathrm{CFE}(\%)=($ the number of colonies $/ 1,000)$ $\mathrm{x} 100$. The colony formation assay was repeated three times. 
Wound healing and Transwell assay. Approximately 6x10 cells/well were incubated in six-well plates. PBS, vector, ad-HepaCAM and ad-HepaCAM-mt were utilized for transfection of LNCaP cells. When the cells reached $\sim 80 \%$ confluency, a straight line was scratched across the cell layer with a pipette tip. Subsequently, fresh culture medium was added, and the cultures were maintained for a further $24 \mathrm{~h}$. The scratch width was measured in micrographs that were captured at 0,12 and $24 \mathrm{~h}$. Approximately $4.0 \times 10^{3}$ cells of each treatment LNCaP culture were inserted into the upper chamber and high glucose DMEM medium containing 30\% FBS was placed in the lower chamber. The LNCaP cells on the upper membrane were swept with swabs after being incubated in serum-free medium for $36 \mathrm{~h}$ at $37^{\circ} \mathrm{C}$. Subsequently, the Transwell membranes were stained with $0.1 \%$ crystal violet solution for $15 \mathrm{~min}$ at room temperature. The LNCaP cells that were anchored to the Transwell membranes were counted using an inverted microscope (Nikon Corporation; magnification, x200). The cell number was quantified from five random fields for each experimental group.

Western blotting. The cells of each treatment group were incubated for $\sim 48 \mathrm{~h}$ at $37^{\circ} \mathrm{C}$, and then, proteins in the cytoplasm and nucleus were extracted using nuclear and cytoplasmic extraction reagents, respectively (Thermo Fisher Scientific, Inc.). Western blotting assays were performed as previously described (8). Briefly, the protein concentration was determined by Enhanced Bicinchoninic Protein Assay kit (Beyotime Institute of Biotechnology, Haimen, China). Protein samples $(50 \mu \mathrm{g})$, stacked by $5 \%$ SDS-PAGE and separated by 10 or $12 \%$ SDS-PAGE were transferred electrophoretically to polyvinylidene fluoride (PVDF) membranes. The PVDF membranes were blocked with $5 \%$ skim milk for $1 \mathrm{~h}$ at $4^{\circ} \mathrm{C}$ and incubated with a primary antibody at $4^{\circ} \mathrm{C}$ overnight. Subsequently, the membrane was incubated with horseradish peroxidase-labelled goat anti-rabbit secondary antibody (1:500; OriGene Technologies, Inc., cat. no. ZF-0316) overnight at $4^{\circ} \mathrm{C}$. The protein bands were visualized by the enhanced chemiluminescent kit (Beyotime Institute of Biotechnology; cat. no. P0018AS) and quantified using Quantity One software 4.6.2 (Bio-Rad Laboratories, Inc., Hercules, CA, USA). The primary antibodies were diluted as follows: Anti-HepaCAM (1:1,000; ProteinTech, cat. no. 18177-1-AP), anti-GAPDH (1:1,000; OriGene Technologies, Inc., cat. no. ZF-0316), anti-histone (1:2,000; Abcam, cat. no. ab176842), anti-AR (1:1,000; ProteinTech, cat. no. 22576-1-AP), anti-c-myc (1:1000; Santa Cruz Biotechnology, Inc., Dallas, TX, USA, cat. no. sc-70469), anti- $\beta$-actin (1:1,000; Abcam, cat. no. ab8226), anti-cyclinD1 (1:1,000; Santa Cruz Biotechnology, Inc., cat. no. sc-70899), anti-E-cadherin (1:2,000; Cell Signaling Technology, Inc., Danvers, MA, USA, cat. no. 3195), anti-N-cadherin (1:2,000; Cell Signaling Technology, Inc., cat. no. 4061), and anti-snail (1:1,000; Cell Signaling Technology, Inc., cat. no. 3895) and anti-Ran (1:1000; Abcam, cat. no. ab157213). All protein expression experiments were repeated three times.

Immunofluorescence. Immunofluorescence was conducted as previously described (22). A total of $1 \times 10^{5}$ cells/well with predetermined treatment were seeded on sterile coverslips at $37^{\circ} \mathrm{C}$ for $\sim 48 \mathrm{~h}$. Cells on the coverslips were fixed in $4 \%$ paraformaldehyde, permeabilized with $0.1 \%$ Triton X-100, and blocked with $5 \%$ fetal bovine serum (Gibco; Thermo Fisher Scientific, Inc.). Subsequently, anti-AR (1:500, ProteinTech, cat. no. 22576-1-AP) or anti-Ran (1:1,000, Abcam, cat. no. ab157213) was added onto the coverslips and they were incubated overnight at $4^{\circ} \mathrm{C}$. The coverslips were then incubated with the secondary antibody for $50 \mathrm{~min}$ at room temperature (1:500, ZSGB; OriGene Technologies, Inc., cat. no. ZF-0316) and the cell nuclei were stained with DAPI (ZSGB; OriGene Technologies, Inc.) for $10 \mathrm{~min}$ at room temperature. The immunofluorescent images were obtained at x400 magnification using a fluorescence microscope (Eclipse 80i; Nikon Corporation, Tokyo, Japan).

Prostate specific antigen (PSA) determination. LNCaP cells $\left(1 \times 10^{5}\right.$ cells/well) were seeded in 96-well plate. PSA protein levels in the supernatant of the LNCaP cells in different treatment groups were determined using solid sandwich ELISA assays using a PSA ELISA kit, according to the manufacturer's protocol (Abcam; cat. no. ab113327).

Reverse transcription-quantitative polymerase chain reaction $(R T-q P C R)$. Cells were seeded in 6-well plates at a density of $2 \times 10^{6}$ cells/well. Total RNA from the LNCaP cells transfected with si-Ran was extracted using a TRIzol kit (Thermo Fisher Scientific, Inc.), and reverse transcription reactions were conducted using the Prime Script RT reagent kit (Takara Biotechnology Co., Ltd.) according to the manufacturer's protocol. RT-qPCR was performed with the SYBR PremixEx Taq $^{\text {TM }}$ II kit (Takara Biotechnology Co., Ltd.) with 400 ng total RNA and oligo (dT) primers in a total $10 \mu \mathrm{l}$. Ran knockdown expression was analyzed by RT-qPCR. The specific primers were as follows: $\beta$-actin forward, 5'-GACCTGTACGCCAAC ACAGT-3', and reverse, 5'-CTCAGGAGGAGCAATGAT CT-3'; Ran forward, 5'-CCAAGGTGGCTACATACTTC-3' and reverse, 5'-TGGTTGGTGATGGTGGTACT-3'. The conditions consisted of predenaturing at $95^{\circ} \mathrm{C}$ for $5 \mathrm{~min}$, followed by 35 cycles of denaturing at $95^{\circ} \mathrm{C}$ for $10 \mathrm{sec}$, annealing at $55^{\circ} \mathrm{C}$ for $50 \mathrm{sec}$, extension at $72^{\circ} \mathrm{C}$ for $1 \mathrm{~min}$, and a final extension at $72^{\circ} \mathrm{C}$ for $5 \mathrm{~min}$. The mRNA expression levels were calculated using the comparative $2^{-\Delta \Delta \mathrm{Cq}}$ method (23) and $\beta$-actin served as a calibrator.

Statistical analysis. Statistical analyses were conducted with SPSS software, version 20.0 (IBM Corp., Armonk, NY, USA). The data are presented as the mean \pm standard deviation. The data between groups was compared using a paired t-test or one-way analysis of variance followed by Tukey's multiple comparisons test where appropriate. The association between the lower HepaCAM expression and higher expression of Ran and AR in PCa was analyzed using Cohen's kappa, and the constituent ratio of positive rate of HepaCAM was analyzed using $\chi^{2}$ tests. $\mathrm{P}<0.05$ was considered to indicate a statistically significant difference.

\section{Results}

HepaCAM is downregulated or absent in PCa tissues and negatively associated with upregulation of Ran and AR. HepaCAM, AR and Ran expression levels were analysed with 
IHC in $46 \mathrm{BPH}$ and PCa tissues. In the IHC analysis, $65.22 \%$ (30/46) of the PCa tissues exhibited negative HepaCAM staining, and $34.78 \%(16 / 46)$ exhibited positive staining (Table I). The association between clinicopathological characteristics and the levels of HepaCAM in the PCa tissues was analysed, and a statistically significant difference was only observed between the expression levels of HepaCAM and the Gleason grade (Table I; $\mathrm{P}<0.05$ ). The semi-quantitative staining scores revealed that the expression levels of HepaCAM were significantly lower in the PCa tissues compared with the $\mathrm{BPH}$ tissues $(\mathrm{P}<0.01$; Fig. $1 \mathrm{~A}$ and $\mathrm{B})$. Additionally, significantly higher expression levels of Ran and AR were detected in the $\mathrm{PCa}$ specimens compared with $\mathrm{BPH}$ specimens $(\mathrm{P}<0.01$; Fig. 1C and D). The association between HepaCAM and AR or Ran was analysed using Cohen's kappa (Table II). Notably, lower HepaCAM expression levels were detected in the samples with higher expression levels of Ran and AR.

Effect of the constructed adenovirus on HepaCAM and HepaCAM-mt protein expression in LNCaP cells. In order to investigate the anti-tumour activity of HepaCAM and its cytoplasmic domain, ad-HepaCAM and ad-HepaCAM-mt were used in LNCaP cells. The GFP protein staining under fluorescence microscope showed high transfection efficiency (data not shown). Furthermore, western blot analysis was used to determine HepaCAM and HepaCAM-mt expression in the LNCaP cells in order to verify transfection efficiency and cell line stability. The results demonstrated that the strong expression of HepaCAM and HepaCAM-mt following transfection was established successfully ( $\mathrm{P}<0.01$; Fig. $2 \mathrm{~A}$ and $\mathrm{B})$.

Effect of HepaCAM and its cytoplasmic domain on cell proliferation and associated proteins in LNCaP cells. Colony formation and MTT assays were used to evaluate whether HepaCAM and its cytoplasmic domain serve an important role in proliferation of LNCaP cells. The results of the colony formation assay revealed similar differences in the viabilities of the cells subjected to the aforementioned treatments (Fig. 2C and D). The MTT analysis revealed that the proliferative capacity of LNCaP cells was suppressed by overexpression of intact HepaCAM. However, overexpression of HepaCAM-mt did not produce inhibiting effects equivalent to those of intact HepaCAM. As shown in Fig. 2E, at day 4, the percentage of viable $\mathrm{LNCaP}$ cells in the ad-HepaCAM group was significantly lower compared with the other three groups $(\mathrm{P}<0.01)$.

In addition, the expression levels of proliferation-associated proteins, including cyclinD1 and c-myc, were significantly reduced after the cells were transfected with ad-HepaCAM compared with the other three groups (Fig. 2F and G). These results suggested that HepaCAM and its cytoplasmic domain serve a key role in the inhibition of cell proliferation and cell growth in PCa. Furthermore, c-myc and cyclin D1 may be associated with the tumour suppression activity of HepaCAM, but this needs to be investigated further.

Effect of HepaCAM and its cytoplasmic domain on migration and invasion-associated proteins in LNCaP cells. The wound healing assays indicated that there was significant suppression of cellular migration in the HepaCAM group compared with the other three groups $(\mathrm{P}<0.01$; Fig. $3 \mathrm{~A}$ and $\mathrm{B})$.
Table I. Association between HepaCAM expression and the clinical characteristics in patients with prostate cancer.

\begin{tabular}{lllll}
\hline & & \multicolumn{2}{c}{ HepaCAM } & \\
\cline { 3 - 3 } Characteristics & Cases (\%) & Negative & Positive & P-value \\
\hline Total no. & $46(100)$ & $30(65.22)$ & $16(34.78)$ & \\
Age & & & & 0.125 \\
$<60$ & $20(43.48)$ & $16(34.78)$ & $4(8.70)$ & \\
$\geq 60$ & $26(56.52)$ & $14(30.43)$ & $12(26.09)$ & \\
Histological stage & & & & 0.729 \\
Ta-T2 & $34(73.91)$ & $22(47.83)$ & $12(26.09)$ & \\
T3-T4 & $12(26.09)$ & $8(17.39)$ & $4(8.70)$ & \\
$\begin{array}{l}\text { Gleason grade } \\
<7\end{array}$ & & & & $0.024^{a}$ \\
$\geq 7$ & $24(52.17)$ & $12(26.09)$ & $12(26.09)$ & \\
\hline
\end{tabular}

${ }^{\mathrm{a}} \mathrm{P}<0.05$. HepaCAM, hepatocyte cell adhesion molecule.

The Transwell experiments also indicated that the migration of LNCaP cells was significantly lower in the HepaCAM group in comparison with the other three groups $(\mathrm{P}<0.01$; Fig. 3C and D). In addition, the E-cadherin expression levels, which are normally absent from LNCaP cells, were slightly higher in the HepaCAM group compared with the other three groups. The levels of N-cadherin and Snail, which are thought to promote migration (24), were significantly attenuated after transfection with ad-HepaCAM compared with the other three groups (Fig. 3E and F). These results indicated that HepaCAM suppresses cell migration via its cytoplasmic domain in vitro. E-cadherin, N-cadherin and Snail maybe involved in the suppressive activity of HepaCAM in LNCaP cells, but this needs to be validated.

Effect of HepaCAM and its cytoplasmic domain on nuclear translocation of AR and Ran. Nuclear translocation of AR is one of the most important steps in AR signalling transduction (25). Ran serves vital roles in modulating the nuclear translocation of proteins (16). To determine whether HepaCAM and its cytoplasmic domain had an impact on the nuclear translocation of Ran and AR, immunofluorescence was employed to assess the subcellular distribution of AR and Ran between the cytoplasm and nucleus (Fig. 4A and B, respectively). The results revealed that AR and Ran expression was upregulated in the cytoplasm and downregulated in the nucleus after cells were transfected with ad-HepaCAM (Fig. 4C). No differences were observed between the HepaCAM-mt group and the blank or vector groups. This suggested that expression of intact HepaCAM altered the distribution of AR and Ran between the cytoplasm and the nucleus and demonstrated that there was a synergic effect between AR and Ran.

Effect of HepaCAM and its cytoplasmic domain on inhibition of PSA in LNCaP cells. The levels of PSA partially reflect the activation of AR targeting genes (26). The analysis of PSA levels revealed that they were significantly lower in the 
A
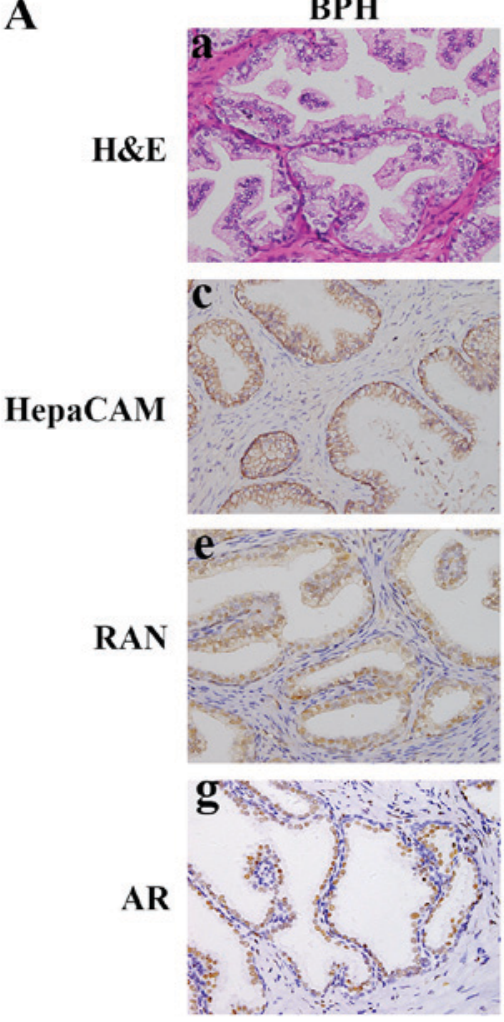
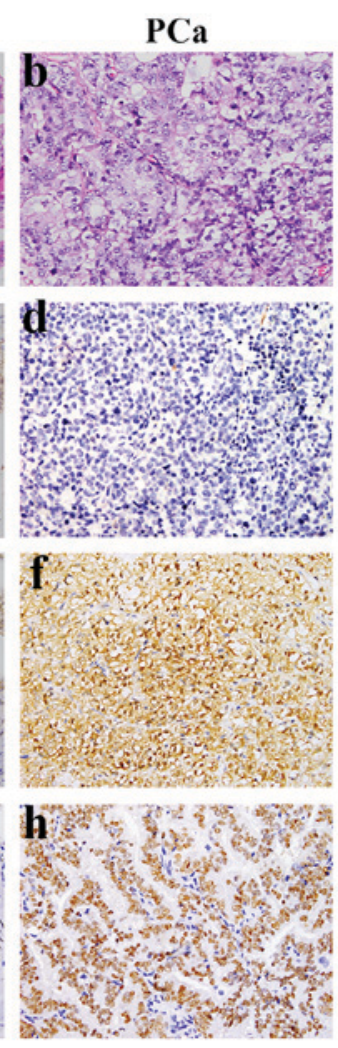

B

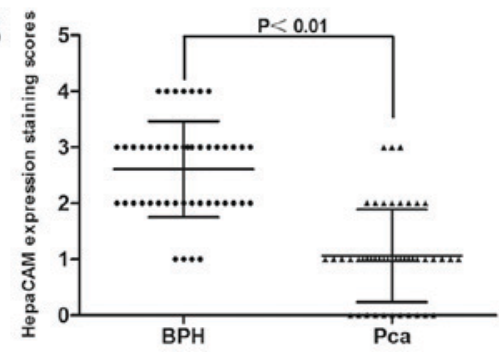

C

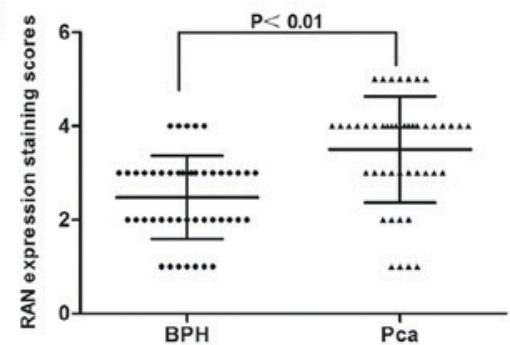

D

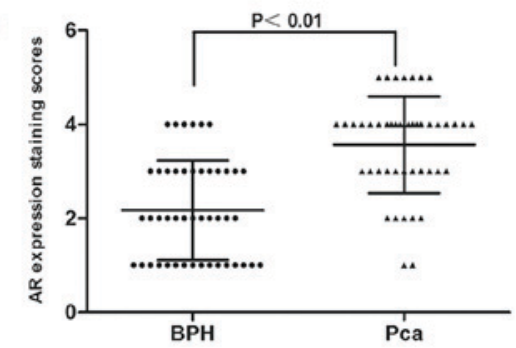

Figure 1. Low expression of HepaCAM is associated with high expression of Ran and AR in PCa tissues. (A) H\&E and immunohistochemical staining in PCa, (a, c, e and g) BPH and (b, d, f and h) PCa. (B) Expression staining scores of HepaCAM, of (C) Ran and of (D) AR in PCa and BPH. Magnification, x400. Ad, adenovirus; AR, androgen receptor; BPH, benign prostatic hyperplasia; HepaCAM, hepatocyte cell adhesion molecule; PCa, prostate cancer.

HepaCAM group compared with the other three groups at 24-48 h. However, following transfection with HepaCAM, no significant difference was identified in the PSA levels between the HepaCAM-mt group and the blank or vector groups at 24-48 h (Fig. 4D). These data suggested that the function of AR may be inhibited by HepaCAM, which maybe via its cytoplasmic domain.

HepaCAM and its cytoplasmic domain affect the nuclear translocation of AR through Ran protein. si-Ran was employed to determine whether Ran serves a role in the nuclear translocation of AR. The results revealed that there was significant downregulation in the total expression of Ran mRNA and protein levels in the si-Ran group compared with the NC group (Fig. 5A-C). This indicates that an active si-Ran was successfully constructed and transfected into the LNCaP cells.

PSA ELISAs were also used to determine the activity of AR. The PSA expression levels were suppressed in the si-Ran, ad-HepaCAM+si-Ran, and ad-HepaCAM-mt+si-Ran groups at 12-48 h compared with the NC group. There was a significant reduction among the si-Ran, ad-HepaCAM+si-Ran and ad-HepaCAM-mt+si-Ran groups at 12-48 h. Additionally, a statistically significant difference was identified between the ad-HepaCAM+si-Ran and ad-HepaCAM-mt+si-Ran groups at 36 or $48 \mathrm{~h}$ (Fig. 5D). This suggested that HepaCAM may inhibited PSA via Ran first. With time, the inhibition efficiency became higher, suggesting that HepaCAM may inhibit PSA in some other way.
As shown in Fig. 5E, when HepaCAM was overexpressed, AR was mainly in the cytoplasm rather than in the nucleus of the LNCaP cells. However, when the cells were treated with ad-HepaCAM-mt, there was a similar distribution of AR in the cytoplasm and the nucleus (Fig. 5F). These data indicate that the cytoplasmic domain regulates AR distribution between the cytoplasm and nucleus. To further investigate whether Ran has an involvement in AR localization, si-Ran was used to transfect the LNCaP cells. Western blotting demonstrated that the levels of AR were higher in the cytoplasm and lower in the nucleus of the si-Ran group. When both ad-HepaCAM and si-Ran were applied, the AR distribution in the cytoplasm and nucleus was similar to that of the si-Ran group (Fig. 5E). The same results were observed when the si-Ran group was compared with the ad-HepaCAM-mt+si-Ran group (Fig. 5F). Therefore, Ran seems to be essential for AR nuclear distribution, and this may be inhibited by HepaCAM through its cytoplasmic domain.

\section{Discussion}

Our previous studies indicated that HepaCAM serves a significant role in various pathophysiological processes in urinary tract cancers, including tumour cell proliferation, apoptosis, migration, and invasion $(7,23,27)$. In the present study, there was a reduction or absence of HepaCAM expression in the majority of the PCa samples. This abnormal expression of HepaCAM was negatively associated with the 
Table II. Association among HepaCAM, Ran and AR expression in PCa tissues.

\begin{tabular}{|c|c|c|c|c|c|c|c|c|}
\hline \multirow[b]{2}{*}{ HepaCAM expression } & \multicolumn{2}{|c|}{ Ran expression } & \multirow[b]{2}{*}{ Kappa } & \multirow[b]{2}{*}{ P-value } & \multicolumn{2}{|c|}{ AR expression } & \multirow[b]{2}{*}{ Kappa } & \multirow[b]{2}{*}{ P-value } \\
\hline & Positive & Negative & & & Positive & Negative & & \\
\hline Negative & 28 & 2 & -0.442 & $\mathrm{P}<0.01^{\mathrm{a}}$ & 29 & 1 & -0.565 & $\mathrm{P}<0.01^{\circ}$ \\
\hline Positive & 6 & 10 & & & 14 & 2 & & \\
\hline
\end{tabular}

${ }^{\mathrm{a}} \mathrm{P}<0.01$. AR, androgen receptor; HepaCAM, hepatocyte cell adhesion molecule; $\mathrm{PCa}$, prostate cancer.

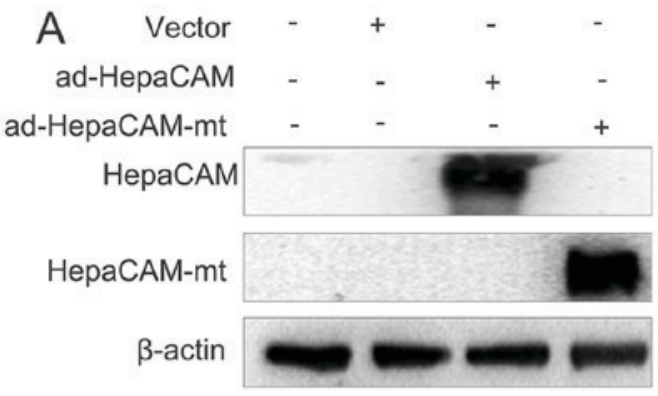

C
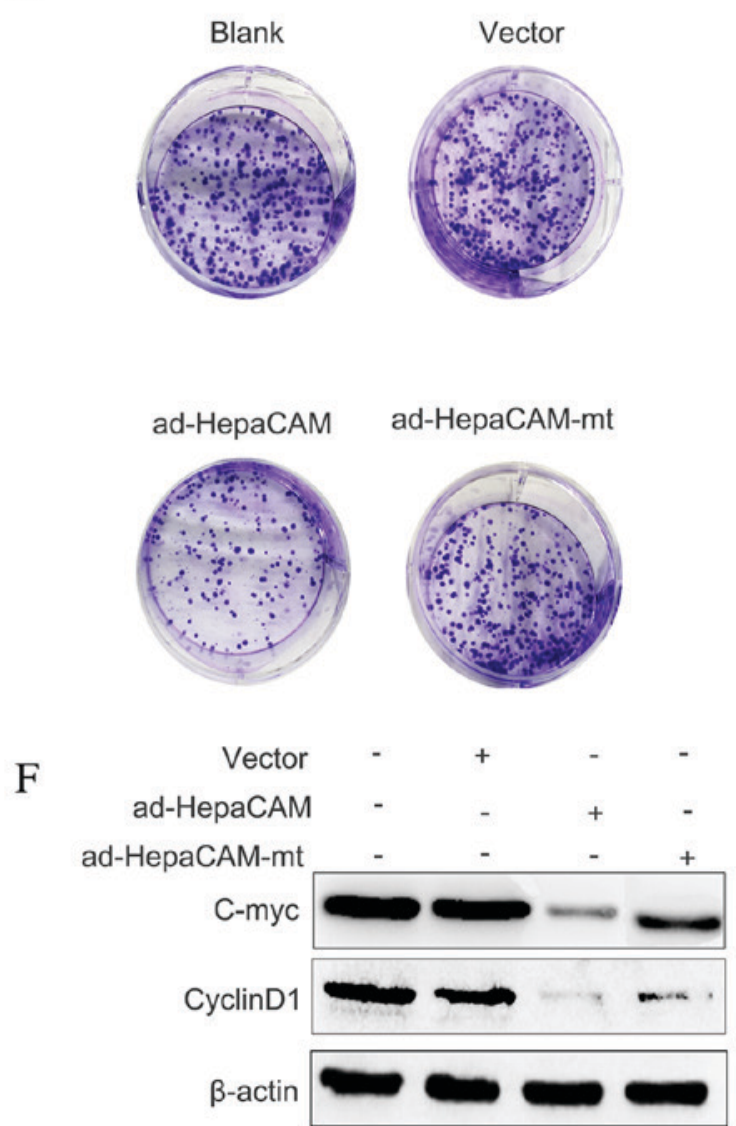

B
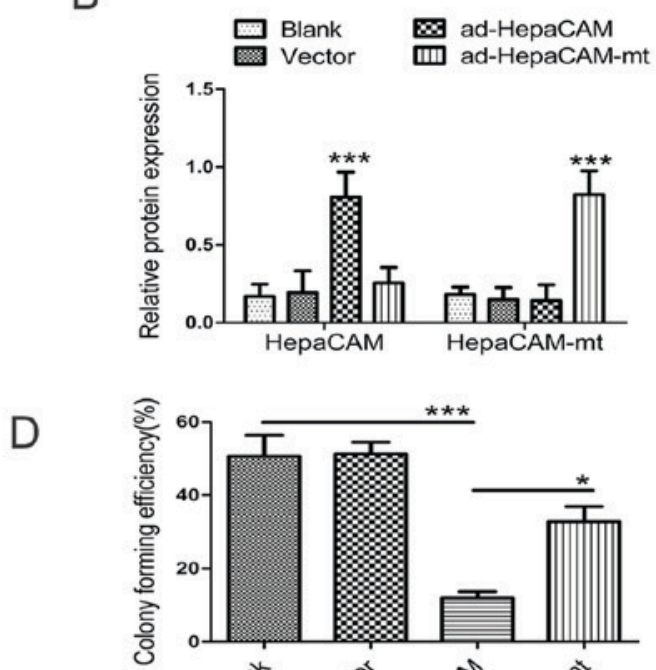

$E$
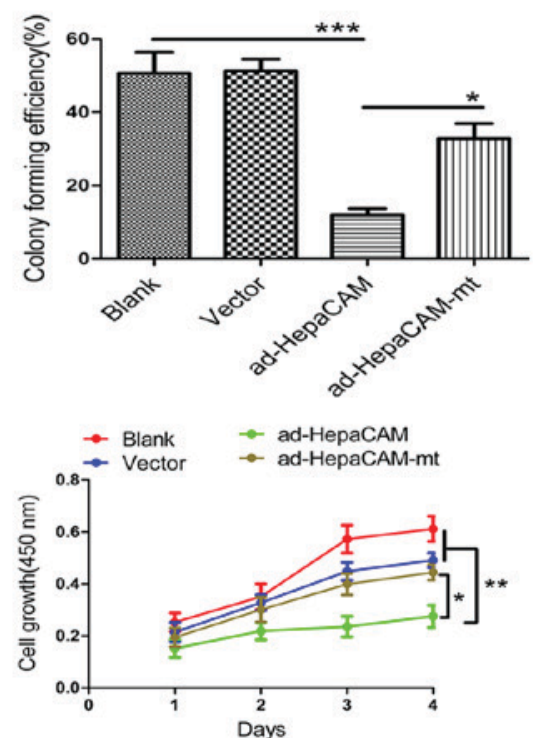

G

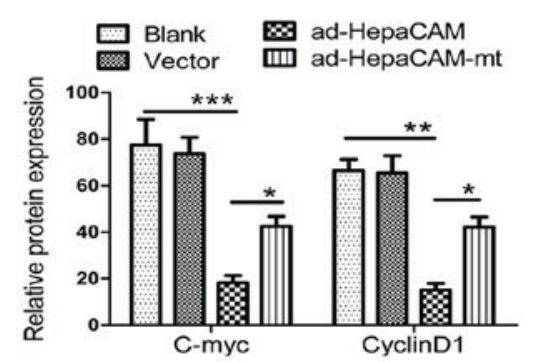

Figure 2. Effect of HepaCAM and its cytoplasmic domain on LNCaP cell proliferation. (A and B) Transfection efficiently of HepaCAM and HepaCAM-mt in LNCaP cells, as measured by western blotting analysis. (C and D) Colony forming efficiency. (E) MTT assays were used to assess the proliferative activity of the LNCaP every day for 4 days. (F) Protein expression levels of c-myc and cyclin D1 and (G) quantification of them, by western blotting. The data are presented as the mean \pm standard deviation. ${ }^{*} \mathrm{P}<0.05 ;{ }^{* *} \mathrm{P}<0.01 ;{ }^{* * *} \mathrm{P}<0.001$. Ad, adenovirus; HepaCAM, hepatocyte cell adhesion molecule; mt, mutant.

Gleason grade, which is associated with high-risk types of $\mathrm{PCa}$ that have a poorer prognosis. Therefore, it is reasonable to presume that HepaCAM may be an inhibiting factor in the progression of PCa. However, the mechanism of the anti-tumour activity of HepaCAM in PCa remains to be elucidated. 
A

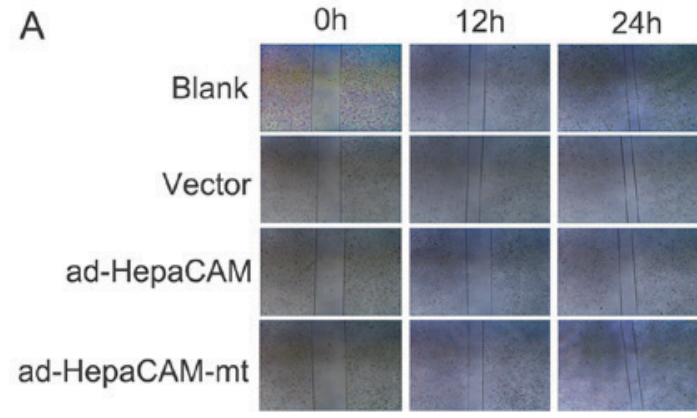

C

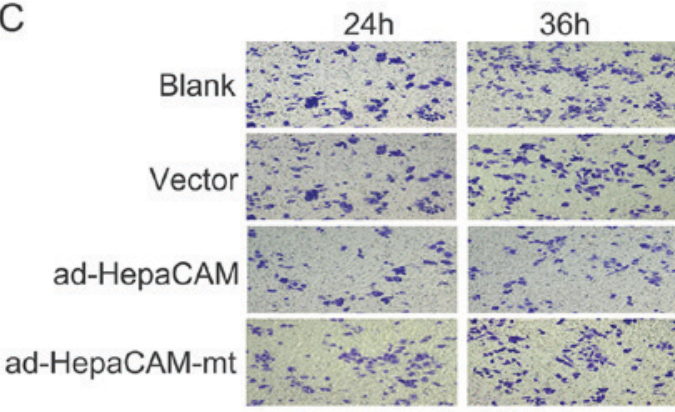

E

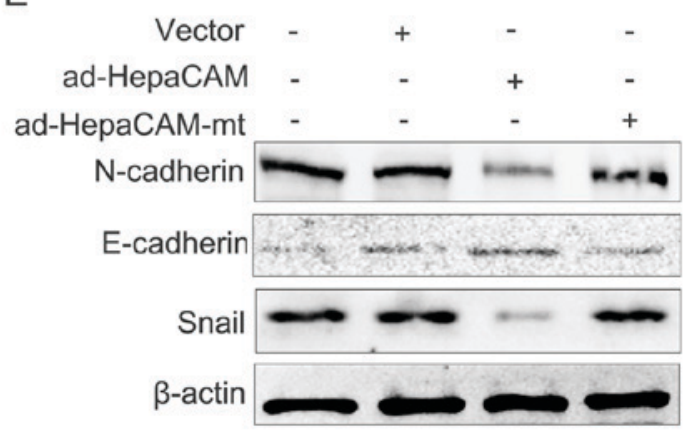

B

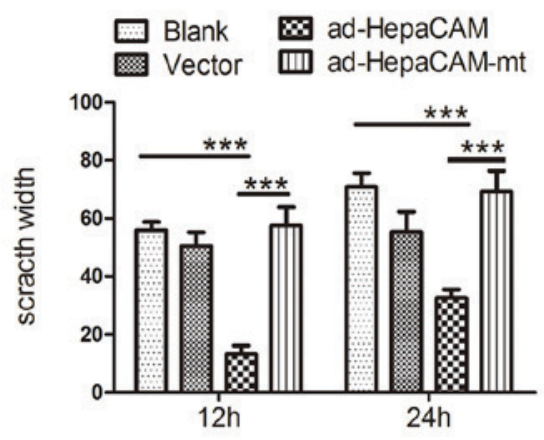

D

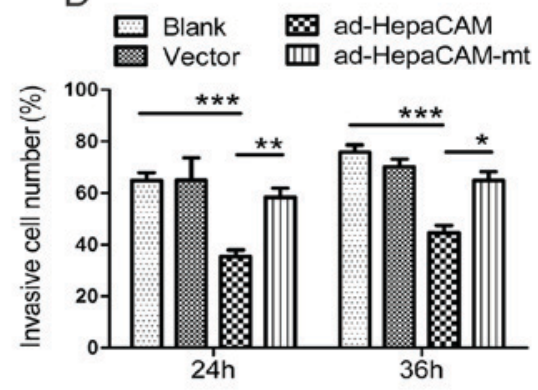

F

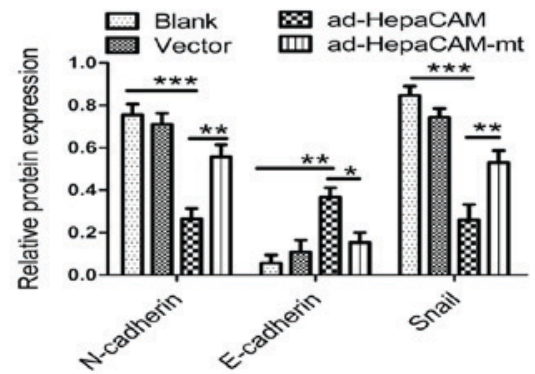

Figure 3. HepaCAM over-expression inhibits invasion and migration of LNCaP cells. (A and B) Wound healing assay of the LNCaP cells with different treatments. (C and D) Migrated LNCaP cells, as measured by Matrigel migration assay. (E and F) Expression levels of E-cadherin, N-cadherin, and Snail, as analysed by western blotting. The data are presented as the mean \pm standard deviation. Magnification, $\mathrm{x} 200 .{ }^{*} \mathrm{P}<0.05,{ }^{* * *} \mathrm{P}<0.01,{ }^{* * * *} \mathrm{P}<0.001$. Ad, adenovirus; HepaCAM, hepatocyte cell adhesion molecule; mt, mutant.

HepaCAM is a transmembrane protein with a phosphorylated cytoplasmic domain (28). In the present study, the function of the cytoplasmic domain of HepaCAM in the inhibition of PCa was investigated. Ad-HepaCAM and ad-HepaCAM-mt were constructed and transfected into PCa cells. The results revealed that overexpression of intact HepaCAM effectively inhibited the growth, proliferation, migration and invasion of cultured LNCaP cells. Furthermore overexpression of intact HepaCAM also downregulated the expression of c-myc and cyclin D1, which are upregulated in the proliferation of LNCaP cells. This downregulation of c-myc and cyclin D1 was also been demonstrated in the colorectal cancer cells (29). In the present study, overexpression of intact HepaCAM was also revealed to downregulate the expression of $\mathrm{N}$-cadherin and Snail proteins, which are upregulated in the migration and invasion of LNCaP cells. This phenomenon has been demonstrated in castration-resistant PCa in a precious study (30). However, overexpression of HepaCAM-mt in the present study did not induce an equivalent anti-tumour effect compared to intact HepaCAM. These data suggest that the anti-tumour activity of HepaCAM in PCa may act through its phosphorylated cytoplasmic domain.

The prostate is a canonical AR-dependent organ, the AR signalling pathway serves a crucial role in the growth and progression of $\mathrm{PCa}$ (31). When androgen binds to the AR in the cytoplasm, the complex is translocated from the cytoplasm to the nucleus, where it targets and promotes downstream gene expression, producing a series of physiological responses (32). In our previous study $(7,20)$, HepaCAM expression significantly reduced the levels of PSA, which is strongly associated with the activation of AR. Further immunofluorescence experiments indicated that overexpression of HepaCAM disrupts the endonuclear and extranuclear distribution of $\mathrm{AR}$ in $\mathrm{LNCaP}$ cells. The accumulation of cytoplasmic AR and reduction of nuclear AR indicated that HepaCAM may inhibit AR nuclear translocation which may inhibit AR signalling pathway. But this needs to be validated. Furthermore, overexpression of HepaCAM-mt did not disturb AR translocation and had no influence on AR distribution, which was similar to the control groups. Therefore, the present study suggested that 


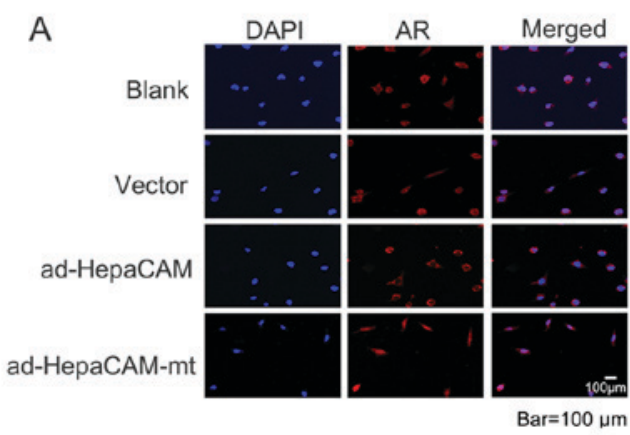

C

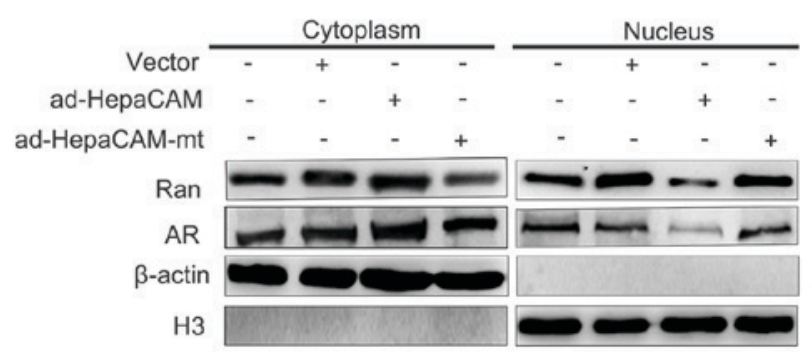

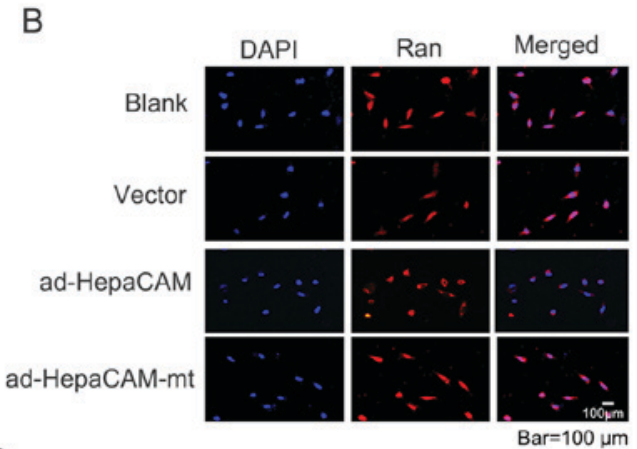

$\mathrm{D}$

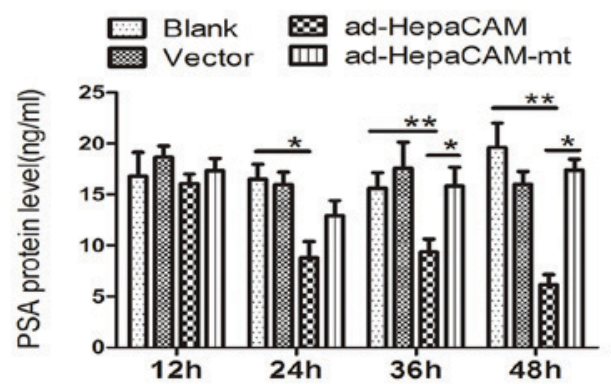

Figure 4. Differences in AR and Ran subcellular localization induced by ad-HepaCAM or ad-HepaCAM-mt in LNCaP cells. AR (A) and Ran (B) intracellular distribution analysis using immunofluorescence staining in the LNCaP cells with different treatments at $48 \mathrm{~h}$. (C) AR and Ran protein expression levels in the cytoplasm and nucleus were detected using western blotting. (D) ELISA analysis of PSA was performed on the culture medium supernatants from different groups at $12-48 \mathrm{~h}$. Magnification, $\mathrm{x} 200$. The data are presented as the mean \pm standard deviation. ${ }^{*} \mathrm{P}<0.05$ and ${ }^{* *} \mathrm{P}<0.01$. Ad, adenovirus; AR, androgen receptor; $\mathrm{H} 3$, histone 3; HepaCAM, hepatocyte cell adhesion molecule; mt, mutant; PSA, prostate specific antigen.
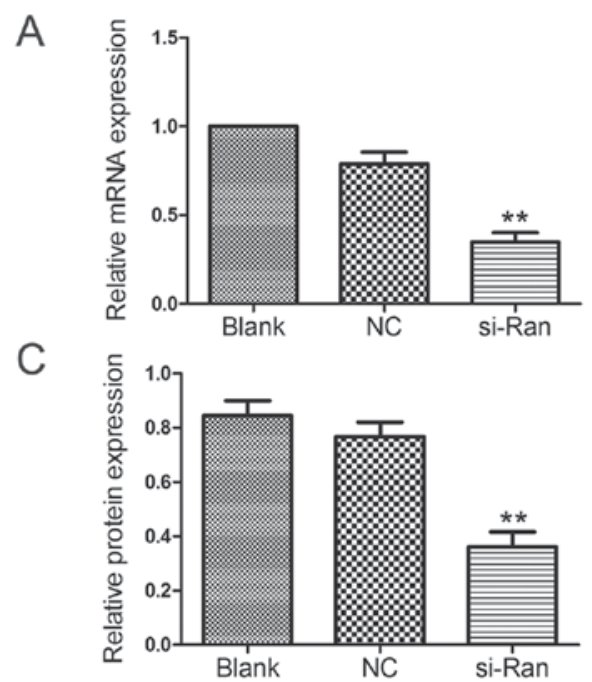

$\mathrm{E}$

B
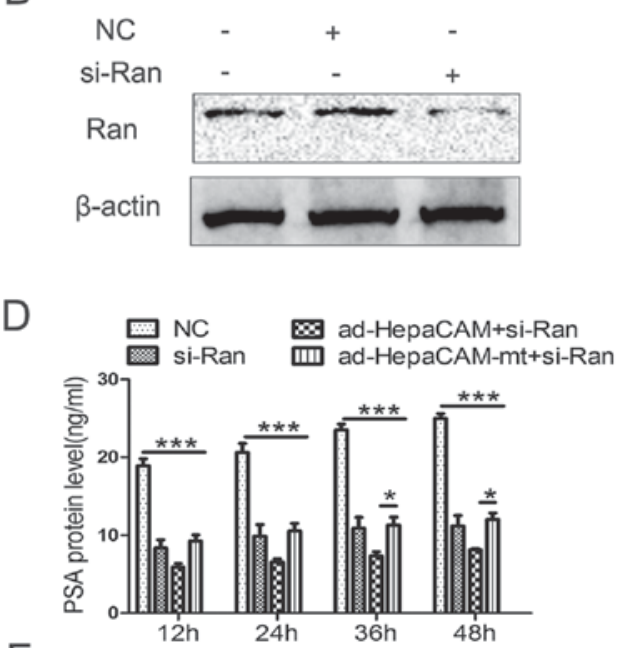

$\mathrm{F}$

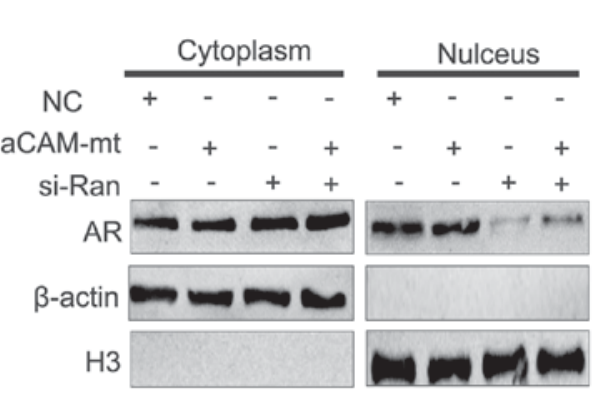

Figure 5. Differences in AR subcellular localization associated with si-Ran. (A) Relative mRNA expression levels of Ran was determined using reverse transcription-quantitative polymerase chain reaction after Ran was silenced by si-Ran in the LNCaP cells. (B and C) Ran protein expression was analysed using western blotting after Ran had been silenced by si-Ran in the LNCaP cells. (D) ELISA analysis of PSA was performed in the culture medium supernatant of the different groups at 12-48 h. Subcellular localization of AR protein in the LNCaP cells treated with si-Ran in the presence or absence of (E) ad-HepaCAM and (F) ad-HepaCAM-mt was analysed using western blotting. The data are presented as the mean \pm standard deviation. ${ }^{*} \mathrm{P}<0.05,{ }^{* * *} \mathrm{P}<0.01$ and ${ }^{* * * *} \mathrm{P}<0.001$. Ad, adenovirus; AR, androgen receptor; HepaCAM, hepatocyte cell adhesion molecule; mt, mutant; NC, negative control; PSA, prostate specific antigen; si, small interference. 
HepaCAM-mediated anti-neoplastic effects were partly caused by the suppression of AR nuclear translocation, which may be accomplished through its phosphorylated cytoplasmic domain.

Energy is consumed when the AR traverses the nuclear pore complex to activate AR signalling (33). This energy is provided by the concentration difference of Ran across the nuclear membrane (34). In the present study overexpression of HepaCAM affected Ran distribution in LNCaP cells. The levels of Ran were higher in the cytoplasm and lower in the nucleus compared with the control cells. By contrast, overexpression of HepaCAM-mt did not result in an altered distribution of Ran, which was similar to the control groups. The differences in Ran distribution were consistent with those of AR distribution in the cytoplasm and nucleus, suggesting that Ran is closely associated with suppression of AR nuclear translocation and subsequent deactivation of the AR signalling pathway. To further investigate the role of Ran in the nuclear translocation of AR, si-Ran was used to silence the expression of Ran. The results revealed that AR was mainly localized in the cytoplasm rather than the nucleus when combined with ad-HepaCAM or ad-HepaCAM-mt. Based on these results, it could be proposed that HepaCAM affects Ran through its cytoplasmic domain and consequently affects AR nuclear translocation, but this needs to be investigated further.

The present study demonstrated that HepaCAM is associated with a reduction in cell proliferation and migration in a PCa cell line. Nuclear translocation of AR and the AR signalling pathway serve a crucial role in the carcinogenesis and tumour development of PCa $(10,11)$. HepaCAM may affect Ran through its cytoplasmic domain and consequently affect AR nuclear translocation. Further studies are required in order to elucidate whether HepaCAM affects the activity of Ran and AR and to determine the binding force between HepaCAM and Ran and AR. Targeting molecules of the HepaCAM/Ran/AR axis may be a potential ADT strategy for PCa.

\section{Acknowledgements}

Not applicable.

\section{Funding}

The present study was supported by the National Natural Science Foundation of China (grant no. 81072086), and the Scientific and Technological Research Program of Chongqing Municipal Education Committee (grant no. KJ110305).

\section{Availability of data and materials}

All data generated or analyzed during the present study are included in this published article.

\section{Authors' contributions}

QD, XW and CL conceived the experiments. QD, ZQ, ZD and WS conducted the experiments and analyzed the data in the researches of the clinical samples. QD, LL and NL conducted the experiments and analyzed the data. QD and XW wrote the manuscript. All authors read and approved the final manuscript.

\section{Ethics approval and consent to participate}

Informed consent forms were signed by all patients, and the study was approved by the Research Ethical Committees of Chongqing Medical University.

\section{Patient consent for publication}

Not applicable.

\section{Competing interests}

The authors declare that they have no competing interests.

\section{References}

1. Siegel R, Ma J, Zou Z and Jemal A: Cancer statistics, 2014. CA Cancer J Clin 64: 9-29, 2014.

2. Chung Moh M, Hoon Lee L and Shen S: Cloning and characterization of hepaCAM, a novel Ig-like cell adhesion molecule suppressed in human hepatocellular carcinoma. J Hepatol 42: 833-841, 2005.

3. Moh MC, Zhang T, Lee LH and Shen S: Expression of hepaCAM is downregulated in cancers and induces senescence-like growth arrest via a p53/p21-dependent pathway in human breast cancer cells. Carcinogenesis 29: 2298-2305, 2008.

4. Xu B, He Y, Wu X, Luo C, Liu A and Zhang J: Exploration of the correlations between interferon- $\gamma$ in patient serum and HEPACAM in bladder transitional cell carcinoma, and the interferon- $\gamma$ mechanism inhibiting BIU-87 proliferation. J Urol 188: 1346-1353, 2012.

5. Xun C, Luo C, Wu X, Zhang Q, Yan L and Shen S: Expression of hepaCAM and its effect on proliferation of tumor cells in renal cell carcinoma. Urology 75: 828-834, 2010.

6. Zhang QL, Luo CL, Wu XH, Wang CY, Xu X, Zhang YY, Liu Q and Shen SL: HepaCAM induces G1 phase arrest and promotes c-Myc degradation in human renal cell carcinoma. J Cell Biochem 112: 2910-2919, 2011.

7. Song X, Wang Y, Du H, Fan Y, Yang X, Wang X, Wu X and Luo C: Overexpression of HepaCAM inhibits cell viability and motility through suppressing nucleus translocation of androgen receptor and ERK signaling in prostate cancer. Prostate 74: 1023-1033, 2014

8. Wang X, Chen E, Tang M, Yang X, Wang Y, Quan Z, Wu X and Luo C: The SMAD2/3 pathway is involved in hepaCAM-induced apoptosis by inhibiting the nuclear translocation of SMAD2/3 in bladder cancer cells. Tumour Biol 37: 10731-10743, 2016.

9. Wen S, Chang HC, Tian J, Shang Z, Niu Y and Chang C: Stromal androgen receptor roles in the development of normal prostate, benign prostate hyperplasia, and prostate cancer. Am J Pathol 185: 293-301, 2015.

10. Scher HI and Sawyers CL: Biology of progressive, castration-resistant prostate cancer: Directed therapies targeting the androgen-receptor signaling axis. J Clin Oncol 23: 8253-8261, 2005.

11. Taplin ME and Balk SP: Androgen receptor: A key molecule in the progression of prostate cancer to hormone independence. J Cell Biochem 91: 483-890, 2004.

12. Chen Y, Clegg NJ and Scher HI: Anti-androgens and androgen-depleting therapies in prostate cancer: New agents for an established target. Lancet Oncol 10: 981-991, 2009.

13. Attard G, Cooper CS and de Bono JS: Steroid hormone receptors in prostate cancer: A hard habit to break? Cancer Cell 16: 458-462, 2009.

14. Dehm SM and Tindall DJ: Androgen receptor structural and functional elements: Role and regulation in prostate cancer. Mol Endocrinol 21: 2855-2863, 2007.

15. Knudsen KE and Scher HI: Starving the addiction: New opportunities for durable suppression of AR signaling in prostate cancer. Clin Cancer Res 15: 4792-4798, 2009.

16. Adam SA and Gerace L: Cytosolic proteins that specifically bind nuclear location signals are receptors for nuclear import. Cell 66: 837-847, 1991. 
17. Rexach M and Blobel G: Protein import into nuclei: Association and dissociation reactions involving transport substrate, transport factors, and nucleoporins. Cell 83: 683-692, 1995.

18. Weis K, Dingwall C and Lamond AI: Characterization of the nuclear protein import mechanism using Ran mutants with altered nucleotide binding specificities. EMBO J 15: 7120-7128, 1996.

19. Sanda MG, Cadeddu JA, Kirkby E, Chen RC, Crispino T, Fontanarosa J, Freedland SJ, Greene K, Klotz LH, Makarov DV, et al: Clinically localized prostate cancer: AUA/ASTRO/SUO Guideline. Part I: Risk stratification, shared decision making, and care options. J Urol 199: 683-690, 2018.

20. Wang Y, Wu X, Ou L, Yang X, Wang X, Tang M, Chen E and Luo C: PLCepsilon knockdown inhibits prostate cancer cell proliferation via suppression of Notch signalling and nuclear translocation of the androgen receptor. Cancer Lett 362: 61-69, 2015.

21. Wang Q, Luo C, Wu X, Du H, Song X and Fan Y: hepaCAM and $\mathrm{p}$-mTOR closely correlate in bladder transitional cell carcinoma and hepaCAM expression inhibits proliferation via an AMPK/mTOR dependent pathway in human bladder cancer cells. J Urol 190: 1912-1918, 2013.

22. Wang L, Hou Y, Sun Y, Zhao L, Tang X, Hu P, Yang J, Zeng Z, Yang G, Cui X and Liu M: c-Ski activates cancer-associated fibroblasts to regulate breast cancer cell invasion. Mol Oncol 7: 1116-1128, 2013

23. Livak KJ and Schmittgen TD: Analysis of relative gene expression data using real-time quantitative PCR and the 2(-Delta Delta C(T)) method. Methods 25: 402-408, 2001.

24. Becker KF, Rosivatz E, Blechschmidt K, Kremmer E, Sarbia M and Höfler H: Analysis of the E-cadherin repressor Snail in primary human cancers. Cells Tissues Organs 185: 204-212, 2007

25. Bennett NC, Gardiner RA, Hooper JD, Johnson DW and Gobe GC: Molecular cell biology of androgen receptor signalling. Int J Biochem Cell Biol 42: 813-827, 2010.

26. Taplin ME and Balk SP: Androgen receptor: A key molecule in the progression of prostate cancer to hormone independence. J Cell Biochem 91: 483-490, 2004.
27. Quan Z,He Y,Luo C, Xia Y, Zhao Y,Liu N and Wu X: Interleukin 6 induces cell proliferation of clear cell renal cell carcinoma by suppressing hepaCAM via the STAT3-dependent up-regulation of DNMT1 or DNMT3b. Cell Signal 32: 48-58, 2017.

28. Moh MC, Zhang C, Luo C, Lee LH and Shen S: Structural and functional analyses of a novel ig-like cell adhesion molecule, hepaCAM, in the human breast carcinoma MCF7 cells. J Biol Chem 280: 27366-27374, 2005.

29. Geng HT, Cao RJ, Cheng L and Liu CY: Overexpression of hepatocyte cell adhesion molecule (hepaCAM) inhibits the proliferation, migration, and invasion in colorectal cancer cells. Oncol Res 25: 1039-1046, 2017.

30. Du Z, Li L, Sun W, Wang X, Zhang Y, Chen Z, Yuan M, Quan Z, Liu N, Hao Y, et al: HepaCAM inhibits the malignant behavior of castration-resistant prostate cancer cells by downregulating Notch signaling and PF-3084014 (a $\gamma$-secretase inhibitor) partly reverses the resistance of refractory prostate cancer to docetaxel and enzalutamide in vitro. Int J Oncol 53: 99-112, 2018.

31. Bluemn EG and Nelson PS: The androgen/androgen receptor axis in prostate cancer. Curr Opin Oncol 24: 251-257, 2012.

32. Ardiani A, Gameiro SR, Kwilas AR, Donahue RN and Hodge JW: Androgen deprivation therapy sensitizes prostate cancer cells to T-cell killing through androgen receptor dependent modulation of the apoptotic pathway. Oncotarget 5: 9335-9348, 2014.

33. Shank LC, Kelley JB, Gioeli D, Yang CS, Spencer A, Allison LA and Paschal BM: Activation of the DNA-dependent protein kinase stimulates nuclear export of the androgen receptor in vitro. J Biol Chem 283: 10568-10580, 2008.

34. Matchett KB, McFarlane S, Hamilton SE, Eltuhamy YS, Davidson MA, Murray JT, Faheem AM and El-Tanani M: Ran GTPase in nuclear envelope formation and cancer metastasis. Adv Exp Med Biol 773: 323-351, 2014.

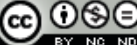

This work is licensed under a Creative Commons
Attribution-NonCommercial-NoDerivatives 4.0 International (CC BY-NC-ND 4.0) License. 